\title{
Analytical Solutions for the Equal Width Equations Containing Generalized Fractional Derivative Using the Efficient Combined Method
}

\author{
Mohammadhossein Derakhshan \\ Department of Industrial Engineering, Apadana Institute of Higher Education, Shiraz, Iran \\ Correspondence should be addressed to Mohammadhossein Derakhshan; m.h.derakhshan.20@gmail.com
}

Received 3 August 2021; Revised 11 September 2021; Accepted 9 November 2021; Published 22 December 2021

Academic Editor: Davood D. Ganji

Copyright ( 2021 Mohammadhossein Derakhshan. This is an open access article distributed under the Creative Commons Attribution License, which permits unrestricted use, distribution, and reproduction in any medium, provided the original work is properly cited.

\begin{abstract}
In this paper, the efficient combined method based on the homotopy perturbation Sadik transform method (HPSTM) is applied to solve the physical and functional equations containing the Caputo-Prabhakar fractional derivative. The mathematical model of this equation of order $\mu \in(0,1]$ with $\lambda \in \mathbb{Z}^{+}, \theta, \sigma \in \mathbb{R}^{+}$is presented as follows: ${ }^{C} \mathfrak{D}_{t}^{\mu} u(x, t)+\theta u^{\lambda}(x, t) u_{x}(x, t)-\sigma u_{x x t}(x, t)=0$, where for $\lambda=1, \theta=1, \sigma=1 s$ and $\lambda=2, \theta=3, \sigma=1$, equations are changed into the equal width and modified equal width equations, respectively. The analytical method which we have used for solving this equation is based on a combination of the homotopy perturbation method and Sadik transform. The convergence and error analysis are discussed in this article. Plots of the analytical results with three examples are presented to show the applicability of this numerical method. Comparison between the obtained absolute errors by the suggested method and other methods is demonstrated.
\end{abstract}

\section{Introduction}

The integrals and derivatives of fractional order in fractional calculus play an important role in many branches such as mathematics, engineering, and physics [1-6]. The differential systems of fractional order are regarded as an extension of the differential systems of integer order and have important application in expressing nonlinear phenomena in many scopes such as applied sciences $[7,8]$, engineering $[9,10]$, and physics $[11,12]$. One of the most important nonlinear differential equations used in many fields of mathematics, engineering, and physics is the equal width (EW) differential equation of fractional order where the equation is a partial differential equation that describes physical behaviors such as water transfer in soils [13], crystal growth [14], and shallow water waves [15]. The main reason for choosing these types of equations is due to their application in mathematics and physics, plasma waves, fluid mechanics, solid-state physics, and chemical physics $[16,17]$. In this paper, we state the time- fractional comprehensive EW equation containing Caputo-Prabhakar fractional derivative as follows [18-20]:

$$
\begin{gathered}
{ }^{C} \mathfrak{D}_{t}^{\mu} u(x, t)+\theta u^{\lambda}(x, t) u_{x}(x, t)-\sigma u_{x x t}(x, t)=0, \\
u(x, 0)=u^{0}(x), u(x, t) \longrightarrow 0, \text { as } x \longrightarrow \pm \infty,
\end{gathered}
$$

where $0<\mu \leq 1, \theta \geq 0, \sigma \geq 0$, and $\lambda \in \mathbb{Z}^{+}$is an integer. For equation (1), we consider two cases as follows:

(1) If $\lambda=1, \theta=1, \sigma=1$ are considered, then equation (1) becomes the nonlinear equal width equation of fractional order $\mu$. This type of equation is one of the most important equations that examine the nonlinear behaviors of physical phenomena such as hydromagnetic waves [21], optics [22], and biological systems [23].

(2) If $\lambda=2, \theta=3, \sigma=1$ are considered, then equation (1) becomes the nonlinear modified equal width equation (MEW) of fractional order $\mu$. 
Moreover, in equation (1), function $u(x, t)$ is the probability density function, $x$ is the spatial coordinate, and $t$ is the temporal coordinate. To find the solutions of these types of equations, the homotopy perturbation Sadik transform method is used, which is a generalization of the homotopy perturbation and Sumudu transform methods. The method presented in this article is similar to the methods discussed in [24-31]. In equation (1), ${ }^{C} \mathfrak{D}_{t}^{\mu}$ is the Caputo-Prabhakar fractional derivative of order $\mu$ which is defined by

$$
\begin{aligned}
{ }^{C} \mathfrak{D}_{t}^{\mu} u(x, t) & =\int_{0}^{t}(t-\tau)^{-\mu} \mathbb{E}_{\rho, 1-\mu}^{-\gamma}\left(\omega(t-\tau)^{\rho}\right) \dot{u}(x, \tau) \mathrm{d} \tau \\
& =\mathfrak{F}^{1-\mu,-\gamma} \frac{d}{\mathrm{~d} t} u(x, t), \quad 0<\mu \leq 1,
\end{aligned}
$$

where $\mathfrak{F}^{1-\mu,-\gamma}$ is the Prabhakar fractional integral and is defined by [32]

$$
\begin{aligned}
\left(\mathfrak{F}^{\mu, \gamma} u\right)(x, t) & =\int_{0}^{t}(t-\tau)^{\mu-1} \mathbb{E}_{\rho, \mu}^{\gamma}\left(\omega(t-\tau)^{\rho}\right) u(x, \tau) \mathrm{d} \tau \\
& =u(x, t) * t^{\mu-1} \mathbb{E}_{\rho, \mu}^{\gamma}\left(\omega t^{\rho}\right)
\end{aligned}
$$

where $u(x, t) \in L^{1}([0, b] \times[0, b]), b \in \mathbb{R}$ and $u(x, t) * t^{\mu-1}$ $\mathbb{E}_{\rho, \mu}^{\gamma}\left(\omega t^{\rho}\right) \in W^{m, 1}([0, b] \times[0, b]), m=\lceil\mu\rceil$ that $W^{m, 1}$ is the Sobolev space. Also, $\mathbb{E}_{\rho, \mu}^{\gamma}$ is the three-parameter MittagLeffler function which is defined by [32]

$$
\mathbb{E}_{\rho, \mu}^{\gamma}(t)=\sum_{n=0}^{\infty} \frac{(\gamma)_{n}}{n ! \Gamma(\rho n+\mu)} t^{n}, \quad \mathfrak{R}(\rho), \mathfrak{R}(\mu)>0, \gamma>0, t \in \mathbb{C},
$$

and $(\gamma)_{n}$ is the Pochhammer symbol which is given by [33]

$$
(\gamma)_{0}=1,(\gamma)_{n}=\gamma(\gamma+1), \ldots,(\gamma+n-1), n \in \mathbb{N} .
$$

The main reason for choosing the three-parameter Mittag-Leffler function in this paper is related to its application in many models such as disordered materials and heterogeneous models [34], Havriliak-Negami models [35, 36], viscoelasticity models [37], stochastic models [38], probability models [39], spherical stellar models [40], Poisson models [41], and fractional models or integral models [42-45]. Many research studies have been conducted in numerical fields to obtain numerical solutions of fractional differential equations such as the Adomian decomposition method [46], the q-homotopy analysis transform method (q-HATM) [47], the Laplace transform method [48], the method based on the implementation of an iterative perturbation method [49], the numerical method based on the Petrov-Galerkin method [50], the Petrov-Galerkin finite element scheme [51], the local discontinuous Galerkin method [52], and other methods $[24,27,30,53]$. Recently, a new integral transform named the Sadik transform was introduced by Sadikali Latif Shaikh in 2018 (see $[54,55]$ ). The Sadik transform is nothing but an unification of the Laplace transform, Sumudu transform, Elzaki transform, and all those integral transforms whose kernels are of exponential type or similar to the kernel of the Laplace transform. The Sadik transform is a very powerful integral transform. In this paper, we apply the HPSTM to solve the nonlinear time-fractional EW equation, MEW equation, and VMEW equation. The key intention of the present work is to extend the utilization of the HPSTM to derive analytical and approximate solutions of the timefractional EW equation, time-fractional MEW equation, and time-fractional VMEW equation.

This paper is organized as follows. Some definitions and mathematical preliminaries of the fractional calculus are presented in Section 2. In Section 3, we introduce an analytical method based on the HPSTM to the nonlinear nonhomogeneous partial differential equation. In Section 4, to show the validity of the suggested method in Section 3, three examples are simulated.

\section{Important Notations}

In this section, we express theorems and lemmas which are used in the next parts.where $\mathfrak{R}(\mu)>0, \mathfrak{R}(\rho)>0$ and $\rho, \mu, \omega, \gamma \in \mathbb{C}$.

Lemma 1. The Laplace transformation of function (4) is given by $[33,56]$

$$
\mathscr{L}\left(t^{\mu-1} \mathbb{E}_{\rho, \mu}^{\gamma}\left(\omega t^{\rho}\right) ; s\right)=s^{-\mu}\left(1-\omega s^{-\rho}\right)^{-\gamma},
$$

Lemma 2 (see [3]). The following relation holds:

$$
\left(\mathfrak{F}^{\mu, \gamma} t^{\nu-1}\right)(t)=\Gamma(\nu) t^{\mu+\nu-1} \mathbb{E}_{\rho, \mu+\nu}^{\gamma}\left(\omega t^{\rho}\right),
$$

where $\mathfrak{R}(\mu)>0, \mathfrak{R}(\rho)>0$ and $\rho, \mu, \omega, \gamma \in \mathbb{C}$.

Lemma 3 (see [57]). Suppose $\Phi_{1}(v, \alpha, \beta)$ is a Sadik transform of $\varphi_{1}(t)$ and $\Phi_{2}(v, \alpha, \beta)$ is a Sadik transform of $\varphi_{2}(t)$. Then, Sadik transform of $\left(\varphi_{1} * \varphi_{2}\right)(t)$ is given by

$$
\mathcal{S}\left[\left(\varphi_{1} * \varphi_{2}\right)(t)\right]=v^{\beta} \Phi_{1}(v, \alpha, \beta) . \Phi_{2}(v, \alpha, \beta)
$$

where * is a convolution. Also, $\Phi_{1}(v, \alpha, \beta)=\mathcal{S}\left(\varphi_{1}(t)\right)=$ $1 / v^{\beta} \int_{0}^{+\infty} e^{-t v^{\alpha}} \varphi_{1}(t)$ and $\Phi_{2}(v, \alpha, \beta)=\mathcal{S}\left(\varphi_{2}(t)\right)=1 / v^{\beta}$ $\int_{0}^{+\infty} e^{-t v^{\alpha}} \varphi_{2}(t)$.

Theorem 1 (see [57]). Suppose $\Phi(v, \alpha, \beta)=\mathcal{S}(\varphi(t))$ and $\varphi(t), \varphi \prime(t), \ldots, \varphi^{m-1}(t), m \in \mathbb{N}$, are continuous. Then,

$$
\mathcal{S}\left[\varphi^{m}(t)\right]=v^{m \alpha} \Phi(v, \alpha, \beta)-\sum_{k=0}^{m-1} v^{k \alpha-\beta} \varphi^{m-k-1}(0) .
$$

Also, the Sadik transform of integration of $\varphi(t)$ is defined by

$$
\mathcal{S}\left[\int_{0}^{t} \varphi(t)\right]=\frac{1}{v^{\alpha}} \Phi(v, \alpha, \beta)
$$

Theorem 2. The Sadik transform of the Caputo-Prabhakar fractional derivative of order $\mu$ for $m=1$ is obtained by 


$$
\begin{aligned}
\mathcal{S}\left({ }^{C} \mathfrak{D}_{t}^{\mu} u(x, t)\right)= & v^{\alpha \mu}\left(1-\omega v^{-\rho \alpha}\right)^{\gamma} U(v, \alpha, \beta) \\
& -v^{\alpha(\mu-1)-\beta}\left(1-\omega v^{-\rho \alpha}\right)^{\gamma} u\left(x, 0^{+}\right),
\end{aligned}
$$

where $U(v, \alpha, \beta)=\mathcal{S}(u(x, t))$ and $\rho, \mu, \omega, \gamma \in \mathbb{C}, \mathfrak{R}(\mu)>0$, $\mathfrak{R}(\rho)>0$.

Proof. By applying equations (2), (6), (8), and (9), we obtain

$$
\begin{aligned}
\mathcal{S}\left({ }^{C} \mathfrak{D}_{t}^{\mu} u(x, t)\right) & =\frac{1}{v^{\beta}} \int_{0}^{\infty} e^{-t v^{\beta}}\left[{ }^{C} \mathfrak{D}_{t}^{\mu} u(x, t)\right] \mathrm{d} t \\
& =\frac{1}{v^{\beta}} \int_{0}^{\infty} e^{-t v^{\beta}}\left\{\int_{0}^{t}(t-\tau)^{-\mu} \mathbb{E}_{\rho, 1-\mu}^{-\gamma}\left(\omega(t-\tau)^{\rho}\right) \frac{d}{\mathrm{~d} \tau} u(x, \tau) \mathrm{d} \tau\right\} \mathrm{d} t \\
& =\frac{1}{v^{\beta}} \int_{0}^{\infty} \int_{\tau}^{\infty} e^{-t v^{\beta}}(t-\tau)^{-\mu} \mathbb{E}_{\rho, 1-\mu}^{-\gamma}\left(\omega(t-\tau)^{\rho}\right) \frac{d}{\mathrm{~d} \tau} u(x, \tau) \mathrm{d} t \mathrm{~d} \tau \\
& =v^{\beta} \mathcal{S}\left(\frac{d}{\mathrm{~d} t} u(x, t)\right) \times \mathcal{S}\left(t^{-\mu} \mathbb{E}_{\rho, 1-\mu}^{-\gamma}\left(\omega t^{\rho}\right)\right) \\
& =\left[v^{\alpha} U(v, \alpha, \beta)-v^{-\beta} u\left(x, 0^{+}\right)\right] \times v^{\alpha \mu-\alpha}\left(1-\omega v^{-\rho \alpha}\right)^{\gamma} \\
& =v^{\alpha \mu}\left(1-\omega v^{-\rho \alpha}\right)^{\gamma} U(v, \alpha, \beta)-v^{\alpha(\mu-1)-\beta}\left(1-\omega v^{-\rho \alpha}\right)^{\gamma} u\left(x, 0^{+}\right) . \\
& \square \quad{ }_{\mathfrak{D}_{t}^{\mu} u(x, t)+\mathbf{A} u(x, t)+\mathbf{N} u(x, t)=g(x, t),} \quad u(x, 0)=u^{0}(x),
\end{aligned}
$$

The proof is proven.

\section{The Proposed Scheme}

This section focuses on an analytical method based on the homotopy perturbation and Sadik transform method for finding the solutions of the following fractional differential equations: where $\mathbf{A} u(x, t)$ is a linear differential operator, $\mathbf{N} u(x, t)$ is a nonlinear differential operator, and $g(x, t)$ is an indicated source term. Using equation (11) and taking the Sadik transform on both sides of equation (13), we get

$$
\begin{gathered}
\mathcal{S}\left({ }^{C} \mathfrak{D}_{t}^{\mu} u(x, t)+\mathbf{A} u(x, t)+\mathbf{N} u(x, t)-g(x, t)\right)=0, \\
v^{\alpha \mu}\left(1-\omega v^{-\rho \alpha}\right)^{\gamma} U(v, \alpha, \beta)-v^{\alpha(\mu-1)-\beta}\left(1-\omega v^{-\rho \alpha}\right)^{\gamma} u\left(x, 0^{+}\right)+\mathcal{S}(\mathbf{A} u(x, t)+\mathbf{N} u(x, t))=\mathcal{S}(g(x, t)), \\
U(v, \alpha, \beta)=v^{-\alpha \mu}\left(1-\omega v^{-\rho \alpha}\right)^{-\gamma} \mathcal{S}(g(x, t))+\frac{1}{v^{\alpha+\beta}} u^{0}(x)-\left(v^{-\alpha \mu}\left(1-\omega v^{-\rho \alpha}\right)^{-\gamma}\right) \times[\mathcal{S}(\mathbf{A} u(x, t)+\mathbf{N} u(x, t))] .
\end{gathered}
$$

Now applying Sadik inverse transform on equation (14), we obtain

$$
\begin{aligned}
u(x, t)= & \mathcal{S}^{-1}\left[v^{-\alpha \mu}\left(1-\omega v^{-\rho \alpha}\right)^{-\gamma} \mathcal{S}(g(x, t))+\frac{1}{v^{\alpha+\beta}} u^{0}(x)\right] \\
& -\mathcal{S}^{-1}\left[\left(v^{-\alpha \mu}\left(1-\omega v^{-\rho \alpha}\right)^{-\gamma}\right)\right. \\
& \times[\mathcal{S}(\mathbf{A} u(x, t)+\mathbf{N} u(x, t))]] .
\end{aligned}
$$

To get the solution of equation (15), we can state the solutions of equation (15) by the following infinite series:

$$
u(x, t)=\sum_{n=0}^{\infty} \varrho^{n} u_{n}(x, t),
$$

where $u_{n}(x, t), n=0,1,2, \ldots$, are known. Also, the nonlinear term $\mathbf{N} u(x, t)$ can be displayed as the following infinite series:

$$
\mathbf{N} u(x, t)=\sum_{n=0}^{\infty} \varrho^{n} \mathbf{H}_{n}(u(x, t)),
$$

where $\mathbf{H}_{n}(u(x, t))=\left\{1 / n !\left(\partial^{n} / \partial \varrho^{n}\right)\left[N\left(\sum_{i=0}^{\infty} \varrho^{i} u_{i}\right)\right]_{\varrho=0}\right\} \quad$ are the Adomian polynomials which were introduced in [46]. Now, substituting equations (16) and (17) into (15), we get 


$$
\begin{aligned}
\sum_{n=0}^{\infty} \varrho^{n} u_{n}(x, t) & =G(x, t)-\mathcal{S}^{-1}\left[\left(v^{-\alpha \mu}\left(1-\omega v^{-\rho \alpha}\right)^{-\gamma}\right) \times\left[\mathcal{S}\left(\mathbf{A} \sum_{n=0}^{\infty} \varrho^{n} u_{n}(x, t)+\mathbf{N} \sum_{n=0}^{\infty} \varrho^{n} u_{n}(x, t)\right)\right]\right] \\
& =G(x, t)-\mathcal{S}^{-1}\left[\left(v^{-\alpha \mu}\left(1-\omega v^{-\rho \alpha}\right)^{-\gamma}\right) \times\left[\mathcal{S}\left(\mathbf{A} \sum_{n=0}^{\infty} \varrho^{n} u_{n}(x, t)+\sum_{n=0}^{\infty} \varrho^{n} \mathbf{H}_{n}(u(x, t))\right)\right]\right]
\end{aligned}
$$

where $\quad G(x, t)=\mathcal{S}^{-1}\left[v^{-\alpha \mu} \quad\left(1-\omega v^{-\rho \alpha}\right)^{-\gamma} \mathcal{S}(g(x, t))+\right.$ $\left.1 / v^{\alpha+\beta} u^{0}(x)\right]$. Therefore, we put the coefficients on powers of $\varrho$ on both sides of relation (18) equally, which results in

$$
\begin{aligned}
\varrho^{0}: u_{0}(x, t) & =G(x, t), \\
\varrho^{1}: u_{1}(x, t) & =-\mathcal{S}^{-1}\left[\left(v^{-\alpha \mu}\left(1-\omega v^{-\rho \alpha}\right)^{-\gamma}\right) \times\left[\mathcal{S}\left(\mathbf{A} u_{0}(x, t)+\mathbf{H}_{0}(u(x, t))\right)\right] ; t\right], \\
\varrho^{2}: u_{2}(x, t) & =-\mathcal{S}^{-1}\left[\left(v^{-\alpha \mu}\left(1-\omega v^{-\rho \alpha}\right)^{-\gamma}\right) \times\left[\mathcal{S}\left(\mathbf{A} u_{1}(x, t)+\mathbf{H}_{1}(u(x, t))\right)\right] ; t\right], \\
& \vdots \\
\varrho^{n+1}: u_{n+1}(x, t) & =-\mathcal{S}^{-1}\left[\left(v^{-\alpha \mu}\left(1-\omega v^{-\rho \alpha}\right)^{-\gamma}\right) \times\left[\mathcal{S}\left(\mathbf{A} u_{n}(x, t)+\mathbf{H}_{n}(u(x, t))\right)\right] ; t\right] .
\end{aligned}
$$

Then, the analytical solution of equation (13) can be obtained as

$$
u(x, t)=\sum_{n=0}^{\infty} u_{n}(x, t)
$$

Theorem 3. Let $u(x, t)$ be the exact solution of equation (13) and $u_{n}(x, t)$ be the approximate solution of equation (13) so that $\left\|u_{n+1}(x, t)\right\| \leq \Phi\left\|u_{n}(x, t)\right\|, \omega \in(0,1), n \in \mathbb{N}$. Then, the series defined by (20) converges.

Proof. From $\left\|u_{n+1}(x, t)\right\| \leq \bowtie\left\|u_{n}(x, t)\right\|$, we obtain

$$
\begin{aligned}
& \left\|u_{1}(x, t)\right\| \leq \Phi\left\|u_{0}(x, t)\right\|=\bowtie\left\|u^{0}(x)\right\|, \\
& \left\|u_{2}(x, t)\right\| \leq \omega\left\|u_{1}(x, t)\right\| \leq \omega \varpi\left\|u^{0}(x)\right\|, \\
& \text { : } \\
& \left\|u_{n+1}(x, t)\right\| \leq \Phi\left\|u_{n}(x, t)\right\| \leq \Phi^{n+1}\left\|u^{0}(x)\right\| .
\end{aligned}
$$

Then,

$$
\sum_{i=n+1}^{\infty}\left\|u_{i}(x, t)\right\| \leq \sum_{i=n+1}^{\infty} \omega^{i}\left\|u^{0}(x)\right\|=\left\|u^{0}(x)\right\| \sum_{i=n+1}^{\infty} \omega^{i} .
$$

Therefore,

$$
\begin{aligned}
\left\|u(x, t)-u_{n+1}(x, t)\right\| & =\left\|\sum_{i=n+1}^{\infty} u_{i}(x, t)\right\| \leq \sum_{i=n+1}^{\infty}\left\|u_{i}(x, t)\right\| \\
& \leq \sum_{i=n+1}^{\infty} \Phi^{i}\left\|u^{0}(x)\right\|=\left\|u^{0}(x)\right\| \sum_{i=n+1}^{\infty} \Phi^{i} \\
& \leq \frac{\Phi^{n+1}}{1-\Phi}\left\|u^{0}(x)\right\| .
\end{aligned}
$$

Because $\omega \in(0,1),\left\|u-u_{n}\right\| \longrightarrow 0$ as $n \longrightarrow \infty$. The proof is proven.

Theorem 4. Let $u(x, t)$ be the exact solution of equation (1) and $(u(x, t))_{N}$ be the best approximate solution of equation (1) so that $\left\|(u(x, t))_{N}^{\lambda+1}-u^{\lambda+1}(x, t)\right\|_{L^{2}(0,1)} \leq \xi \|(u(x, t))_{N}$ $-u(x, t) \|_{L^{2}(0,1)}, \xi \in(0,1), \lambda \in \mathbb{Z}^{+}$. Then, the following relation for the error holds:

$$
\begin{aligned}
& \|\mathbf{E}(u(x, t))\|_{L^{2}(0,1)} \leq\left[k_{1}\left(t^{1-\mu} \mathbb{E}_{\rho, 2-\mu}^{-\gamma}\left(\omega t^{\rho}\right)\right)+\frac{\theta}{\lambda+1} k_{2} \xi+\sigma k_{1} k_{2}^{2}\right] c N^{-\sigma} 2^{-k \sigma}\left\|u^{(\sigma)}\right\|_{L^{2}(0,1)}, \\
& \|\mathbf{E}(u(x, t))\|_{L^{2}(0,1)} \leq\left[k_{1}\left(t^{1-\mu} \mathbb{E}_{\rho, 2-\mu}^{-\gamma}\left(\omega t^{\rho}\right)\right)+\frac{\theta}{\lambda+1} k_{2} \xi+\sigma k_{1} k_{2}^{2}\right] c N^{2 q-(1 / 2)-\sigma} 2^{k(q-\sigma)}\left\|u^{(\sigma)}\right\|_{L^{2}(0,1)}, \quad q \geq 1 .
\end{aligned}
$$


Proof. Since $u(x, t)$ is the exact solution of equation (1) and $(u(x, t))_{N}$ is the best approximate solution of equation (1), then we have

$$
\begin{aligned}
\|\mathbf{E}(u(x, t))\|_{L^{2}(0,1)}= & \left.\left.\|{ }^{C} \mathfrak{D}_{t}^{\mu}(u(x, t))_{N}+\theta(u(x, t))_{N}^{\lambda}\left((u(x, t))_{N}\right)_{x}-\sigma(u(x, t))\right)_{N}\right)_{x x t} \\
& -{ }^{C} \mathfrak{D}_{t}^{\mu}(u(x, t))+\theta u^{\lambda}(x, t) u_{x}(x, t)-\sigma u_{x x t}(x, t) \|_{L^{2}(0,1)} \\
= & \|{ }^{C} \mathfrak{D}_{t}^{\mu}\left[(u(x, t))_{N}-u(x, t)\right]+\theta\left[(u(x, t))_{N}^{\lambda}\left((u(x, t))_{N}\right)_{x}-u^{\lambda}(x, t) u_{x}(x, t)\right] \\
& -\sigma\left[\left((u(x, t))_{N}\right)_{x x t}-u_{x x t}(x, t)\right] \|_{L^{2}(0,1)} \\
= & \|{ }^{C} \mathfrak{D}_{t}^{\mu}\left[(u(x, t))_{N}-u(x, t)\right]+\frac{\theta}{\lambda+1}\left(\frac{\partial}{\partial x}\left((u(x, t))_{N}^{\lambda+1}-u^{\lambda+1}(x, t)\right)\right. \\
& \left.-\sigma\left[\frac{\partial^{3}}{\partial x^{2} \partial t}\left((u(x, t))_{N}\right)-u(x, t)\right)\right] \| \\
\leq & \left\|{ }^{C} \mathfrak{D}_{t}^{\mu}\left[(u(x, t))_{N}-u(x, t)\right]_{L^{2}(0,1)}+\frac{\theta}{\lambda+1}\right\|\left(\frac{\partial}{\partial x}\left((u(x, t))_{N}^{\lambda+1}-u^{\lambda+1}(x, t)\right) \|_{L^{2}(0,1)}\right. \\
& \left.+\sigma \|\left[\frac{\partial^{3}}{\partial x^{2} \partial t}\left((u(x, t))_{N}\right)-u(x, t)\right)\right] \|
\end{aligned}
$$

Using Lemma 2, equation (3), and $\left\|f_{1} * f_{2}\right\|_{L^{2}(0,1)} \leq$ $\left\|f_{1}\right\|_{1}\left\|f_{2}\right\|_{L^{2}(0,1)}, f_{1} \in L^{1}(0,1), f_{2} \in L^{2}(0,1), \quad$ for $\quad \| C \mathfrak{D}_{t}^{\mu}$ $\left[(u(x, t))_{N}-u(x, t)\right] \|_{L^{2}(0,1)}$, we get

$$
\begin{aligned}
\left\|C \mathfrak{D}_{t}^{\mu}\left[(u(x, t))_{N}-u(x, t)\right]\right\|_{L^{2}(0,1)}^{2} & =\left\|\mathfrak{F}^{1-\mu,-\gamma}\left((u(x, t))_{N}-u(x, t)\right)\right\|_{L^{2}(0,1)}^{2} \\
& =\left\|\mathfrak{F}^{1-\mu,-\gamma} \frac{d}{\mathrm{~d} t}\left((u(x, t))_{N}-u(x, t)\right)\right\|_{L^{2}(0,1)}^{2}=\left\|t^{-\mu} \mathbb{E}_{\rho, 1-\mu}^{-\gamma}\left(\omega t^{\rho}\right) * \frac{d}{\mathrm{~d} t}\left((u(x, t))_{N}-u(x, t)\right)\right\|_{L^{2}(0,1)}^{2} \\
& \leq\left\|t^{-\mu} \mathbb{E}_{\rho, 1-\mu}^{-\gamma}\left(\omega t^{\rho}\right)\right\|_{1} \times\left\|\frac{d}{\mathrm{~d} t}\left((u(x, t))_{N}-u(x, t)\right)\right\|_{L^{2}(0,1)}^{2}
\end{aligned}
$$

and by using the Lipschitz condition with constant $0<k_{1}<1$ for derivative of the first order with respect to the variable $t$, we have

$$
\begin{aligned}
& \left\|{ }^{C} \mathfrak{D}_{t}^{\mu}\left[(u(x, t))_{N}-u(x, t)\right]\right\|_{L^{2}(0,1)}^{2} \leq k_{1}^{2}\left(t^{1-\mu} \mathbb{E}_{\rho, 2-\mu}^{-\gamma}\left(\omega t^{\rho}\right)\right)^{2}\left\|(u(x, t))_{N}-u(x, t)\right\|_{L^{2}(0,1)}^{2}, \\
& \left\|^{C} \mathfrak{D}_{t}^{\mu}\left[(u(x, t))_{N}-u(x, t)\right]\right\|_{L^{2}(0,1)}^{2} \leq k_{1}\left(t^{1-\mu} \mathbb{E}_{\rho, 2-\mu}^{-\gamma}\left(\omega t^{\rho}\right)\right)\left\|(u(x, t))_{N}-u(x, t)\right\|_{L^{2}(0,1)} .
\end{aligned}
$$


By using the Lipschitz condition with constant $0<k_{2}<1$ for derivative of the first order with respect to the variable $x$ and theorem assumptions, from equation (25), we obtain

$$
\begin{aligned}
\|\mathbf{E}(u(x, t))\|_{L^{2}(0,1)} \leq & k_{1}\left(t^{1-\mu} \mathbb{E}_{\rho, 2-\mu}^{-\gamma}\left(\omega t^{\rho}\right)\right)\left\|(u(x, t))_{N}-u(x, t)\right\|_{L^{2}(0,1)}+\frac{\theta}{\lambda+1} k_{2} \xi\left\|(u(x, t))_{N}-u(x, t)\right\|_{L^{2}(0,1)} \\
& +\sigma k_{1} k_{2}^{2}\left\|(u(x, t))_{N}-u(x, t)\right\|_{L^{2}(0,1)}
\end{aligned}
$$

and according to Theorem 2 introduced in [58], we have

$$
\begin{aligned}
\|\mathbf{E}(u(x, t))\|_{L^{2}(0,1)} & \leq\left[k_{1}\left(t^{1-\mu} \mathbb{E}_{\rho, 2-\mu}^{-\gamma}\left(\omega t^{\rho}\right)\right)+\frac{\theta}{\lambda+1} k_{2} \xi+\sigma k_{1} k_{2}^{2}\right]\left\|(u(x, t))_{N}-u(x, t)\right\|_{L^{2}(0,1)} \\
& \leq\left[k_{1}\left(t^{1-\mu} \mathbb{E}_{\rho, 2-\mu}^{-\gamma}\left(\omega t^{\rho}\right)\right)+\frac{\theta}{\lambda+1} k_{2} \xi+\sigma k_{1} k_{2}^{2}\right] c N^{-\sigma} 2^{-k \sigma}\left\|u^{(\sigma)}\right\|_{L^{2}(0,1)}, \\
\| \mathbf{E}\left(u((x, t)) \|_{L^{2}(0,1)}\right. & \leq\left[k_{1}\left(t^{1-\mu} \mathbb{E}_{\rho, 2-\mu}^{-\gamma}\left(\omega t^{\rho}\right)\right)+\frac{\theta}{\lambda+1} k_{2} \xi+\sigma k_{1} k_{2}^{2}\right] c N^{2 q-(1 / 2)-\sigma} 2^{k(q-\sigma)}\left\|_{u^{(\sigma)}}\right\|_{L^{2}(0,1)}, \quad q \geq 1,
\end{aligned}
$$

where $u(x, t),(u(x, t))_{N} \in \mathfrak{H}^{\sigma}(0,1), \sigma \geq 0, \quad \xi>0, \quad$ that $\mathfrak{S}^{\sigma}(0,1), \sigma \geq 0$, given [58].

\section{Numerical Results}

In this section, we show the approximate solution obtained by the proposed method which is named the homotopy perturbation Sadik transform method with three examples to express its performance. In this section, we consider the absolute error as follows:

$$
\mathbf{E}(x, t)=\left|u(x, t)-u_{n}(x, t)\right|
$$

where $u(x, t)$ is the exact solution of equation (1) and $u_{n}(x, t)$ is the numerical solution of equation (1).
4.1. Example 1. We consider the following fractional EW equation with $\lambda=1, \theta=1, \sigma=1$ :

$$
\begin{gathered}
{ }^{C} \mathfrak{D}_{t}^{\mu} u(x, t) u(x, t)+u(x, t) u_{x}(x, t) \\
-u_{x x t}(x, t)=0, \quad 0<\mu \leq 1, \\
\cdot u(x, 0)=3 \sec h^{2}\left(\frac{x-15}{2}\right),
\end{gathered}
$$

when $\quad \gamma=0$, and the exact solution is $u(x, t)=3 \sec h^{2}(x-15-t / 2)$. By applying the proposed method given in Section 3, we solve equation (31); then,

$$
\begin{aligned}
\sum_{n=0}^{\infty} \varrho^{n} u_{n}(x, t)= & 3 \sec h^{2}\left(\frac{x-15}{2}\right)+\mathcal{S}^{-1}\left[\left(v^{-\alpha \mu}\left(1-\omega v^{-\rho \alpha}\right)^{-\gamma}\right)\right. \\
& \left.\times\left[\mathcal{S}\left(\left(\sum_{n=0}^{\infty} \varrho^{n} u_{n}(x, t)\right)_{x x t}-\sum_{n=0}^{\infty} \varrho^{n} \mathbf{H}_{n}(u(x, t))\right)\right] ; t\right]
\end{aligned}
$$

where $\mathbf{H}_{n}(u(x, t))$ is the nonlinear $u u_{x}$. The polynomials $\mathbf{H}_{n}(u(x, t))$ are calculated as follows:

$$
\begin{aligned}
& \mathbf{H}_{0}(u(x, t))=u_{0}\left(u_{0}\right)_{x}, \\
& \mathbf{H}_{1}(u(x, t))=u_{0}\left(u_{1}\right)_{x}+u_{1}\left(u_{0}\right)_{x},
\end{aligned}
$$

Comparing coefficients of same powers of $p$ in equation (32), we obtain

$$
\begin{aligned}
\varrho^{0}: u_{0}(x, t)= & 3 \sec h^{2}\left(\frac{x-15}{2}\right) \\
\varrho^{1}: u_{1}(x, t)= & \mathcal{S}^{-1}\left[\left(v^{-\alpha \mu}\left(1-\omega v^{-\rho \alpha}\right)^{-\gamma}\right)\right. \\
& \left.\times\left[\mathcal{S}\left(\left(u_{0}(x, t)\right)_{x x t}-\mathbf{H}_{0}(u(x, t))\right)\right] ; t\right] \\
= & 9 \sec h^{4}\left(\frac{x-15}{2}\right) \tanh \left(\frac{x-15}{2}\right) t^{\mu} \mathbb{E}_{\rho, 1+\mu}^{\gamma}\left(\omega t^{\rho}\right),
\end{aligned}
$$



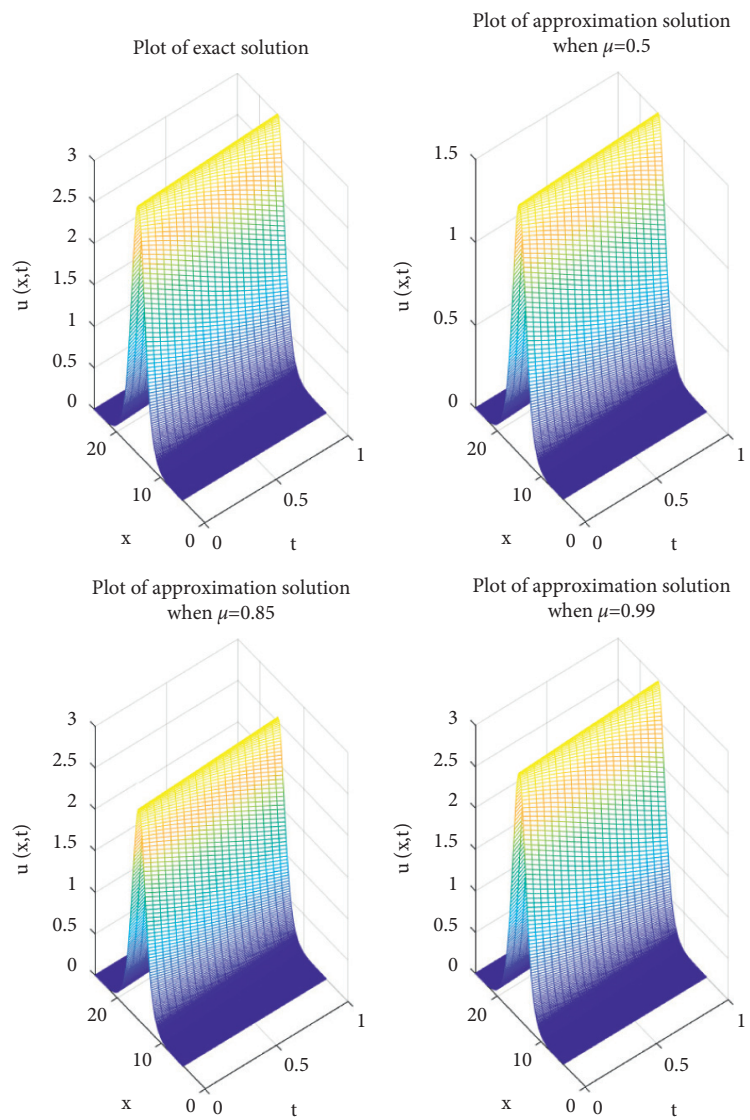

Plot of approximation solution when $\mu=0.99$

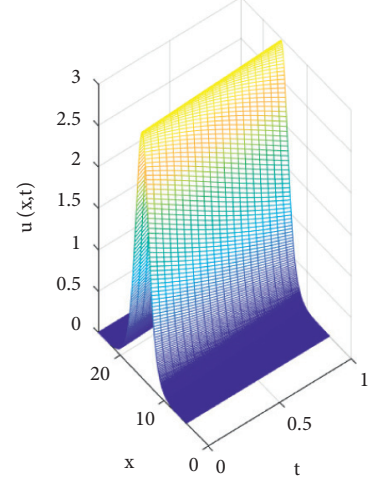

Plot of error

when $\mu=0.5$

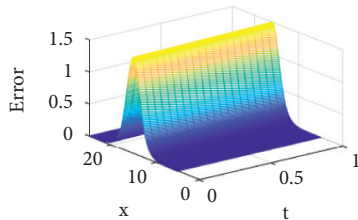

Plot of error when $\mu=0.85$

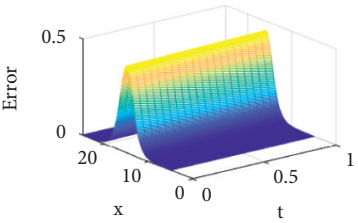

Plot of error

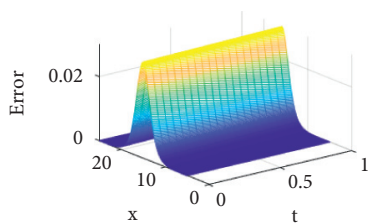

FIGURE 1: Graphs of approximate solution and the exact solution and absolute error for different values of $\mu$ when $\mu=0.5,0.85,0.99,1$ and $\rho=\omega=\gamma=1$ for example 1 .

Then, the approximate solution of $u(x, t)$ is obtained as

$$
\begin{aligned}
u(x, t) & =\sum_{n=0}^{\infty} u_{n}(x, t) \\
& =3 \sec h^{2}\left(\frac{x-15}{2}\right)+9 \sec h^{4}\left(\frac{x-15}{2}\right) \tanh \left(\frac{x-15}{2}\right) t^{\mu} \mathbb{E}_{\rho, 1+\mu}^{\gamma}\left(\omega t^{\rho}\right)+\ldots
\end{aligned}
$$




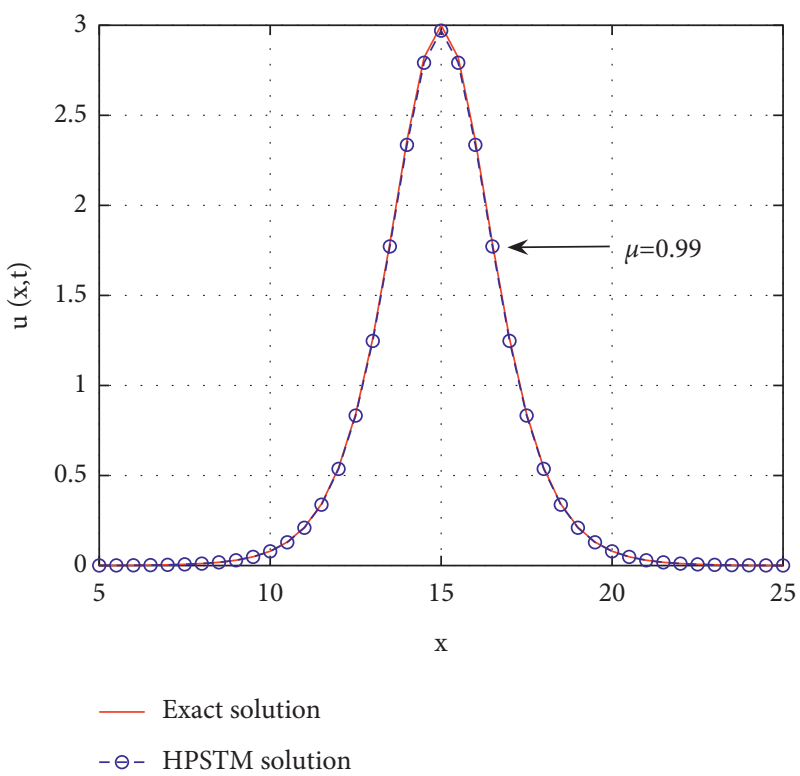

FIgURE 2: Comparison of exact solution and approximate solution with homotopy perturbation Sadik transform method when $t=0.0001$ and $\rho=\omega=\gamma=1$ for example 1 .

TABLE 1: Comparison between the absolute error in [2] and the absolute error for homotopy perturbation Sadik transform method with different values of $\mu$ for example 1 .

\begin{tabular}{cccrr}
\hline$x$ & & $\left|u-u_{n}\right|$ & & $\left|u-u_{n}\right|$ \\
& $\mu=0.5$ & $\mu=0.85$ & $\mu=0.99$ & $7.242560880 E-06$ \\
10 & $1.4006135487 E-09$ & $1.4210717446 E-09$ & $1.4412222851 E-09$ & $1.610108943 E-05$ \\
11 & $1.4804436993 E-09$ & $1.4994344786 E-09$ & $1.5179577058 E-09$ & $2.246615682 E-05$ \\
12 & $1.5534369379 E-09$ & $1.5703105528 E-09$ & $1.5865522230 E-09$ & $1.6444028957 E-09$ \\
13 & $1.16169787676 E-09$ & $1.6310851118 E-09$ & $1.6890873673 E-09$ & $1.494373852 E-05$ \\
14 & $1.16685291313 E-09$ & $1.6792699469 E-09$ & $1.7314989455 E-09$ & $7.000000000 E-09$ \\
15 & $1.17271757893 E-09$ & $1.7298751108 E-09$ & $1.7271887190 E-09$ & $1.482115835 E-04$ \\
16 & $1.7315032733 E-09$ & $1.7298837530 E-09$ & $1.7058693781 E-09$ & $2.493848058 E-05$ \\
17 & $1.7186088774 E-09$ & $1.7127505267 E-09$ & $1.6685738938 E-09$ & $2.247007139 E-05$ \\
18 & $1.16891247436 E-09$ & $1.6793110759 E-09$ & $1.6170367143 E-09$ & $1.610299997 E-05$ \\
19 & $1.6444545342 E-09$ & $1.6311399784 E-09$ & $1.5535056371 E-09$ & $7.243321962 E-06$ \\
20 & $1.5866158664 E-09$ & $1.5703768054 E-09$ & & \\
\hline
\end{tabular}

The comparison between the exact and the approximate solutions and the absolute error are expressed in Figure 1 for different values of $\mu$ when $\mu=0.5,0.85,0.99,1$. Also, in Figure 2, the exact and the numerical solutions at $t=0.0001$ are given. In Table 1, a comparison between the absolute error in [2] and the absolute error for HPSTM with different values of $\mu$ is given.

4.2. Example 2. Consider the following MEW equation with $\lambda=2, \theta=3, \sigma=1$ :

$$
\begin{gathered}
{ }^{C} \mathfrak{D}_{t}^{\mu} u(x, t) u(x, t)+3 u^{2}(x, t) u_{x}(x, t) \\
-u_{x x t}(x, t)=0, \quad 0<\mu \leq 1 \\
\cdot u(x, 0)=\frac{1}{4} \sec h(x-30)
\end{gathered}
$$

for $\gamma=0$, and the exact solution is $u(x, t)=1 / 4 \mathrm{sec} h$ $(x-30-t / 4)$. Applying the HPSTM on equation (36), we obtain 

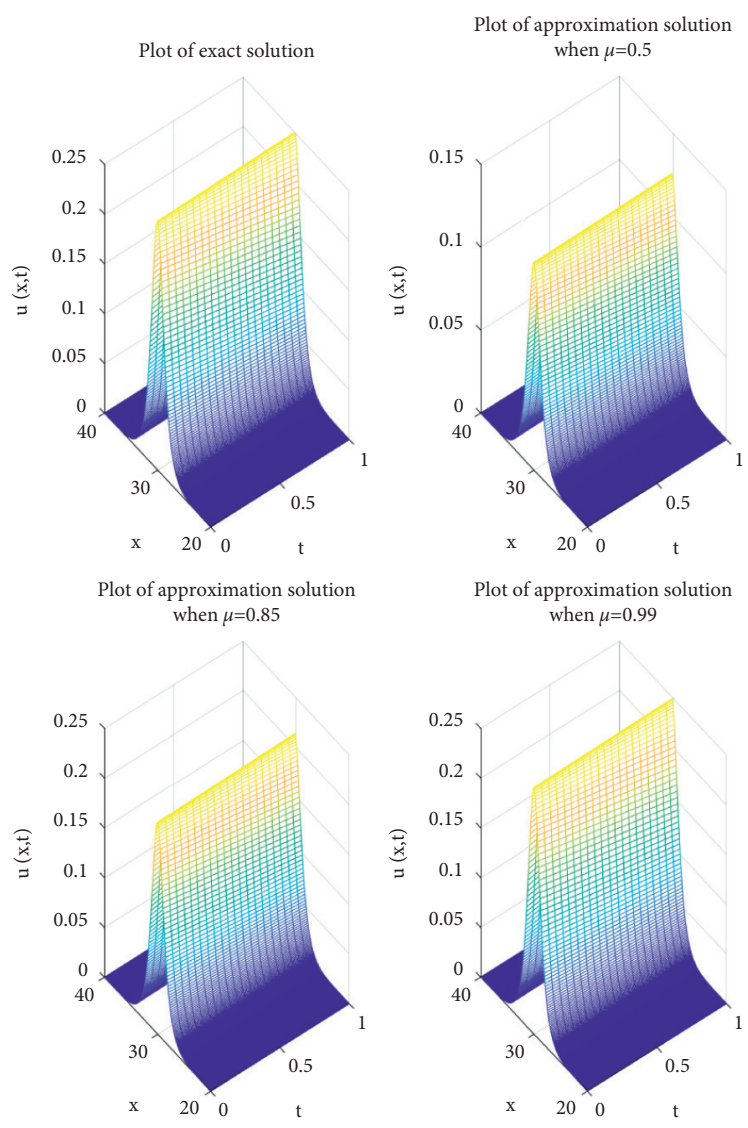

Plot of approximation solution

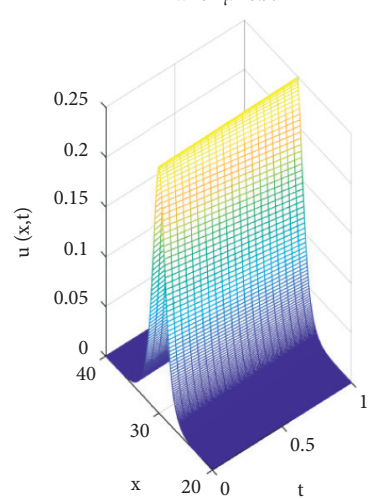

Plot of error

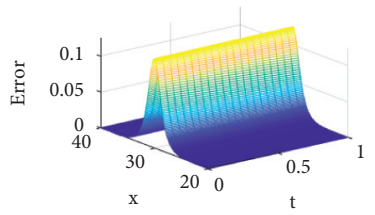

Plot of error

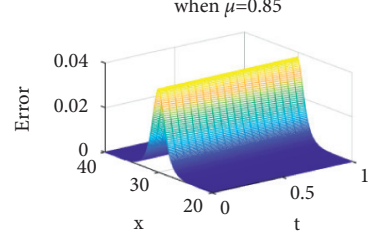

Plot of error

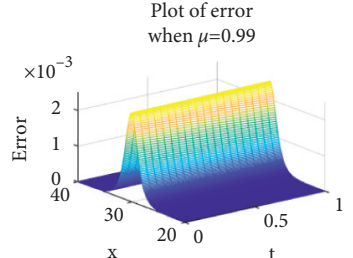

FIGURE 3: Graphs of the approximation and exact solutions and absolute error for different values of $\mu$ when $\mu=0.5,0.85,0.99,1$ and $\rho=\omega=\gamma=1$ for example 2 .

$$
\begin{aligned}
\sum_{n=0}^{\infty} \varrho^{n} u_{n}(x, t)= & \frac{1}{4} \sec h(x-30)+\mathcal{S}^{-1}\left[\left(v^{-\alpha \mu}\left(1-\omega v^{-\rho \alpha}\right)^{-\gamma}\right)\right. \\
& \left.\times\left[\mathcal{S}\left(\left(\sum_{n=0}^{\infty} \varrho^{n} u_{n}(x, t)\right)_{x x t}-\sum_{n=0}^{\infty} \varrho^{n} \mathbf{H}_{n}(u(x, t))\right)\right] ; t\right],
\end{aligned}
$$




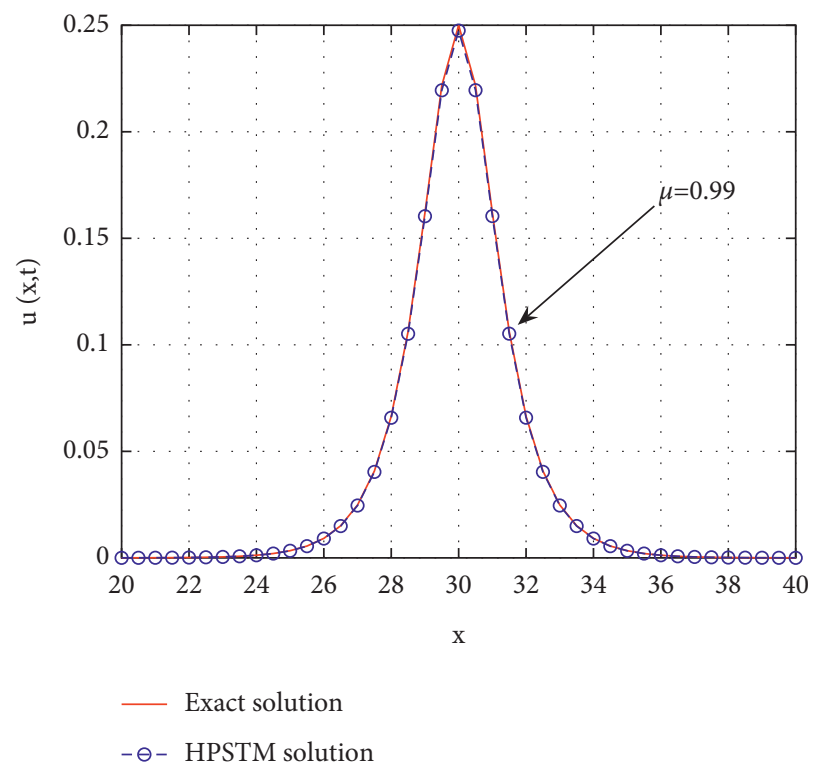

FIGURE 4: Comparison of exact solution and approximate solution with homotopy perturbation Sadik transform method when $t=0.0001$ and $\rho=\omega=\gamma=1$ for example 2 .

TABLE 2: Comparison between the absolute error in [2] and the absolute error for homotopy perturbation Sadik transform method with different values of $\mu$ for example 2 .

\begin{tabular}{ccccr}
\hline$x$ & $\mu=0.5$ & $\left|u-u_{n}\right|$ & $\mu$ & $\left|u-u_{n}\right|$ \\
& $\mu=0.85$ & $\mu=0.99$ & $8.419085488 E-07$ \\
\hline 25 & $4.9233966126 E-09$ & $4.8905216623 E-09$ & $4.8523133049 E-09$ & $2.284567360 E-06$ \\
26 & $4.7611171679 E-09$ & $4.7088017083 E-09$ & $4.6524848045 E-09$ & $6.130812328 E-06$ \\
27 & $4.5293288600 E-09$ & $4.4632329663 E-09$ & $4.3946122324 E-09$ & $1.516464963 E-05$ \\
28 & $4.2511941226 E-09$ & $4.1770584430 E-09$ & $4.1017168885 E-09$ & $2.113002231 E-05$ \\
29 & $3.9485345529 E-09$ & $3.8712015990 E-09$ & $3.7936781419 E-09$ & $7.800000000 E-09$ \\
30 & $3.6388500689 E-09$ & $3.5618921913 E-09$ & $3.4854390699 E-09$ & $2.113176846 E-05$ \\
31 & $3.3345479504 E-09$ & $3.2603221763 E-09$ & $3.1870278987 E-09$ & $1.516821359 E-05$ \\
32 & $3.04351478950 E-09$ & $2.9734094032 E-09$ & $2.9044644353 E-09$ & $6.132358065 E-06$ \\
33 & $2.7701849765 E-09$ & $2.7048973756 E-09$ & $2.6408655090 E-09$ & $2.285133776 E-06$ \\
34 & $2.5166006751 E-09$ & $2.4563726541 E-09$ & $2.3974113232 E-09$ & $8.421208912 E-07$ \\
35 & $2.2832615675 E-09$ & $2.2280532855 E-09$ & $2.1740726737 E-09$ & \\
\hline
\end{tabular}

where the polynomials $\mathbf{H}_{n}(u(x, t))$ are represented as the nonlinear terms which are denoted as

$$
\sum_{n=0}^{\infty} \varrho^{n} \mathbf{H}_{n}(u(x, t))=3 u^{2} u_{x} .
$$

The polynomials $\mathbf{H}_{n}(u(x, t))$ are calculated as follows:

$$
\begin{aligned}
\mathbf{H}_{0}(u(x, t)) & =3 u_{0}^{2}\left(u_{0}\right)_{x}, \\
\mathbf{H}_{1}(u(x, t)) & =3 u_{0}^{2}\left(u_{1}\right)_{x}+6 u_{0} u_{1}\left(u_{0}\right)_{x}, \\
& \vdots
\end{aligned}
$$

Comparing coefficients of same powers of $p$ in equation (37), we obtain

$$
\begin{aligned}
\varrho^{0}: u_{0}(x, t)= & \frac{1}{4} \sec h(x-30), \\
\varrho^{1}: u_{1}(x, t)= & \mathcal{S}^{-1}\left[\left(v^{-\alpha \mu}\left(1-\omega v^{-\rho \alpha}\right)^{-\gamma}\right)\right. \\
& \left.\times\left[\mathcal{S}\left(\left(u_{0}(x, t)\right)_{x x t}-\mathbf{H}_{0}(u(x, t))\right)\right] ; t\right] \\
= & \frac{3}{64} \sec h^{3}(x-30) \tanh (x-30) t^{\mu} \mathbb{E}_{\rho, 1+\mu}^{\gamma}\left(\omega t^{\rho}\right), \\
\vdots & .
\end{aligned}
$$

Finally, the approximate solution of $u(x, t)$ is given by 

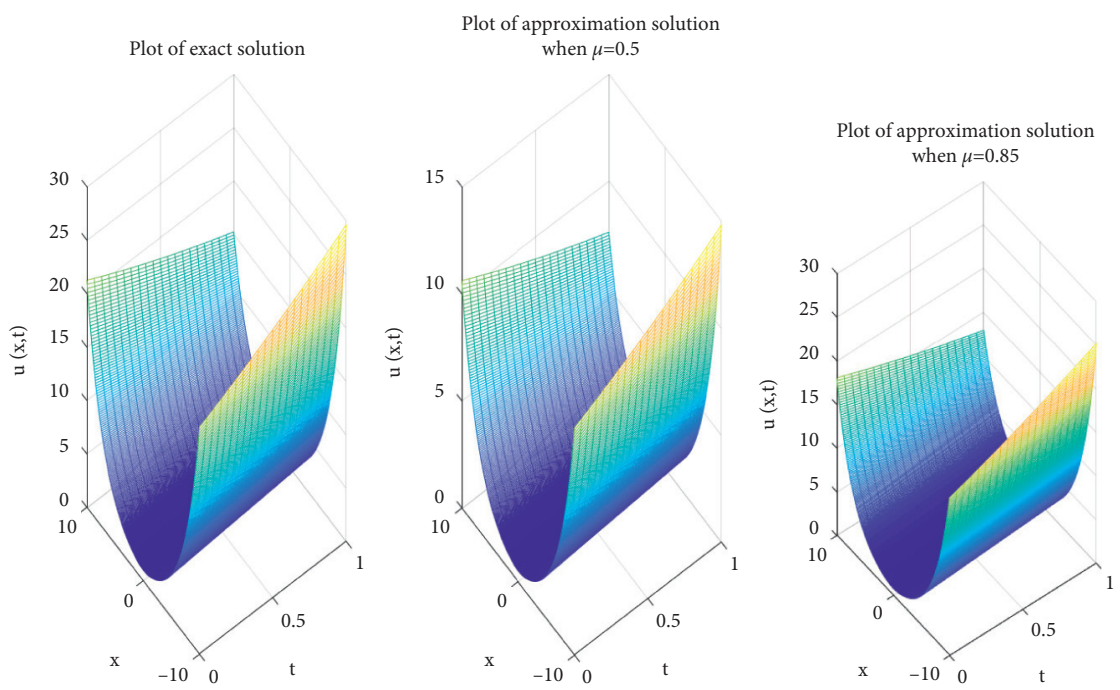

Plot of approximation solution

Plot of error

when $\mu=0.5$
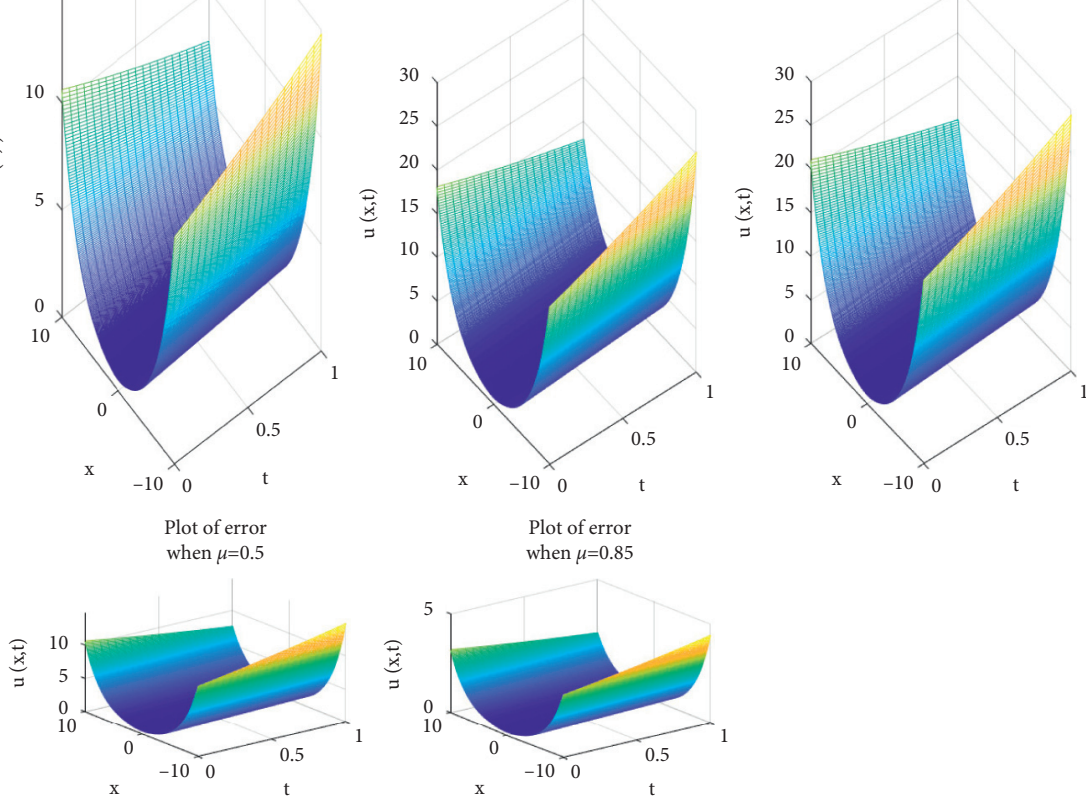

Plot of error

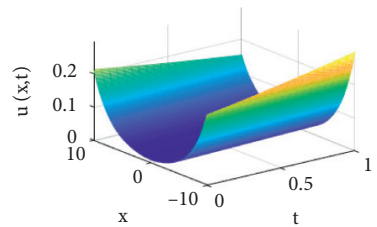

FIGURE 5: Graphs of the approximation and exact solutions and the absolute error for different values of $\mu$ when $\mu=0.5,0.85,0.99,1$ and $\rho=\omega=\gamma=1$ for example 3 .

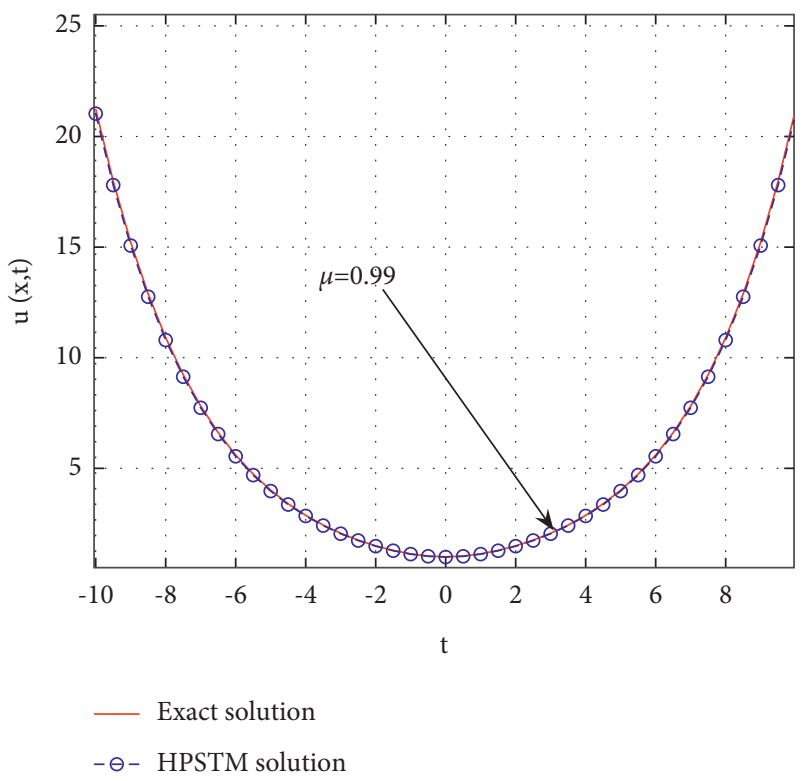

FIgURE 6: Comparison of exact solution and approximate solution with homotopy perturbation and Sadik transform method when $t=$ 0.0001 and $\rho=\omega=\gamma=1$ for example 3 . 
TABLE 3: Comparison between the absolute error in [2] and the absolute error for homotopy perturbation Sadik transform method with different values of $\mu$ for example 3 .

\begin{tabular}{|c|c|c|c|c|}
\hline \multirow{2}{*}{$x$} & \multicolumn{2}{|c|}{$\left|u-u_{n}\right|$} & \multicolumn{2}{|c|}{$\left|u-u_{n}\right|$} \\
\hline & $\mu=0.5$ & $\mu=0.85$ & $\mu=0.99$ & Method [2] \\
\hline 0 & $4.5709477859 E-09$ & $4.5330148328 E-09$ & $4.4953966746 E-09$ & $1.000000000 E-09$ \\
\hline 1 & $4.4210943150 E-09$ & $4.3844049537 E-09$ & $4.3480200672 E-09$ & $2.563406968 E-05$ \\
\hline 2 & $4.2761536328 E-09$ & $4.2406670942 E-09$ & $4.2054750487 E-09$ & $4.601380631 E-05$ \\
\hline 3 & $4.1359646818 E-09$ & $4.1016415335 E-09$ & $4.0676032238 E-09$ & $6.668778565 E-05$ \\
\hline 4 & $4.0003716853 E-09$ & $3.9671737878 E-09$ & $3.9342513912 E-09$ & $9.224742588 E-05$ \\
\hline 5 & $3.8692239749 E-09$ & $3.8371144396 E-09$ & $3.8052713736 E-09$ & $1.244701205 E-04$ \\
\hline 6 & $3.7423758233 E-09$ & $3.7113189714 E-09$ & $3.6805198536 E-09$ & $1.615598280 E-04$ \\
\hline 7 & $3.6196862832 E-09$ & $3.5896476064 E-09$ & $3.5598582149 E-09$ & $1.923074240 E-04$ \\
\hline 8 & $3.5010190312 E-09$ & $3.4719651533 E-09$ & $3.4431523893 E-09$ & $1.781977647 E-04$ \\
\hline 9 & $3.3862422172 E-09$ & $3.3581408576 E-09$ & $3.3302727086 E-09$ & $3.522674800 E-06$ \\
\hline 10 & $3.2752283194 E-09$ & $3.2480482574 E-09$ & $3.2210937625 E-09$ & $6.615336660 E-04$ \\
\hline
\end{tabular}

$$
\begin{aligned}
u(x, t)= & \sum_{n=0}^{\infty} u_{n}(x, t) \\
= & \frac{1}{4} \sec h(x-30)+\frac{3}{64} \sec h^{3}(x-30) \tanh \\
& (x-30) t^{\mu} E_{\rho, 1+\mu}^{\gamma}\left(\omega t^{\rho}\right)+\ldots
\end{aligned}
$$

The comparison between exact solution and the approximate solution and absolute error are expressed in Figure 3 for different values of $\mu$ when $\mu=0.5,0.85,0.99,1$. Figure 4 shows the comparison of exact solution with homotopy perturbation Sadik transform method solution at $t=0.0001$. In Table 2, a comparison between the absolute error in [2] and the absolute error for HPSTM with different values of $\mu$ is given.

In the example 2, we study the solution of a variant of fractional modified equal width equation (VMEW) which is obtained by the proposed method.

4.3. Example 3. Consider the following VMEW equation:

$$
\begin{gathered}
{ }^{C} \mathfrak{D}_{t}^{\mu} u(x, t) u(x, t)+\frac{12}{7} u_{x}^{6}(x, t) \\
-\frac{3}{7} u_{x x t}^{6}(x, t)=0, \quad 0<\mu \leq 1, \\
\cdot u(x, 0)=\cosh ^{5 / 2}\left(\frac{5 x}{6}\right),
\end{gathered}
$$

and the exact solution is $u(x, t)=\cosh ^{5 / 2}(5(x-t) / 6)$ when $\gamma=0$. Applying the homotopy perturbation and Sadik transform method on equation (42), we have

$$
\begin{aligned}
\sum_{n=0}^{\infty} \varrho^{n} u_{n}(x, t)= & \cosh ^{5 / 2}\left(\frac{5 x}{6}\right)+\mathcal{S}^{-1}\left[\left(v^{-\alpha \mu}\left(1-\omega v^{-\rho \alpha}\right)^{-\gamma}\right)\right. \\
& \left.\times\left[\mathcal{S}\left(\sum_{n=0}^{\infty} \varrho^{n} \mathbf{H}_{n}(u(x, t))\right)\right] ; t\right],
\end{aligned}
$$

where $\mathbf{H}_{n}(u(x, t))$ are obtained as

$$
\sum_{n=0}^{\infty} \varrho^{n} \mathbf{H}_{n}(u(x, t))=\frac{3}{7} u_{x x t}^{6}-\frac{12}{7} u_{x}^{6},
$$

and $\mathbf{H}_{0}(u(x, t)), \ldots, \mathbf{H}_{n}(u(x, t))$ are calculated as follows:

$$
\mathbf{H}_{0}(u(x, t))=\frac{3}{7}\left(u_{0}^{6}\right)_{x x t}-\frac{12}{7}\left(u_{0}^{6}\right)_{x}
$$

$$
\vdots
$$

Comparing coefficients of same powers of $p$ in equation (43), we obtain

$$
\begin{aligned}
& \begin{aligned}
\varrho^{0}: u_{0}(x, t)= & \cosh ^{5 / 2}\left(\frac{5 x}{6}\right), \\
\varrho^{1}: u_{1}(x, t)= & \mathcal{S}^{-1}\left[\left(v^{-\alpha \mu}\left(1-\omega v^{-\rho \alpha}\right)^{-\gamma}\right)\right. \\
& \left.\times\left[\mathcal{S}\left(\mathbf{H}_{0}(u(x, t))\right)\right] ; t\right] \\
= & -\frac{24}{7} \cosh ^{7 / 5}\left(\frac{5 x}{6}\right) \sinh \left(\frac{5 x}{6}\right) t^{\mu} \mathbb{E}_{\rho, 1+\mu}^{\gamma}\left(\omega t^{\rho}\right), \\
\vdots & \cdot
\end{aligned} \\
& \text { So, the approximate } \operatorname{solution} \text { of } u(x, t) \text { is calculated as } \\
& \begin{array}{c}
u(x, t)=\sum_{n=0}^{\infty} u_{n}(x, t) \\
=\cosh ^{5 / 2}\left(\frac{5 x}{6}\right)-\frac{24}{7} \cosh \frac{7}{5}\left(\frac{5 x}{6}\right) \sinh \left(\frac{5 x}{6}\right) t^{\mu} \mathbb{E}_{\rho, 1+\mu}^{\gamma}\left(\omega t^{\rho}\right)+\cdots
\end{array}
\end{aligned}
$$

Comparison between the exact and the approximate solutions and the absolute error are shown in Figure 5 for different values of $\mu$ when $\mu=0.5,0.85,0.99,1$. Also, in Figure 6, comparison of the exact solution and the approximate solution at $t=0.0001$ is illustrated. In Table 3, a comparison between the absolute error in [2] and the absolute error for homotopy perturbation Sadik transform method with different values of $\mu$ is given. 


\section{Conclusion}

This paper describes a numerical method based on the homotopy perturbation Sadik transform method for solving the equations such as EW, MEW, and VMEW. The fractional derivatives are used in this paper in the CaputoPrabhakar sense. This paper demonstrates that the HPSTM is sufficient, easy, and suitable for various other nonlinear models. It can be seen that as $x$ increases, it leads to variation in $u(x, t)$ for waves in plasma at specific value of $t$. All tables and figures show that the proposed method produces numerical solutions with more accuracy. Comparison between the obtained absolute errors by the suggested method and method presented in [2] is demonstrated. Discussion on the convergence and error analysis of the proposed method is presented. Three numerical examples are given to show the applicability of the suggested method.

\section{Data Availability}

No data were used to support this study.

\section{Conflicts of Interest}

The author declares that there are no conflicts of interest.

\section{References}

[1] K. Diethelm, D. Baleanu, and E. Scalas, Fractional Calculus: Models and Numerical Methods, World Scientific, Singapore, 2012.

[2] A. Goswami, J. Singh, D. Kumar, and Sushila, "An efficient analytical approach for fractional equal width equations describing hydro-magnetic waves in cold plasma," Physica A: Statistical Mechanics and its Applications, vol. 524, pp. 563$575,2019$.

[3] A. A. Kilbas, M. Saigo, and R. K. Saxena, "Generalized MittagLeffler function and generalized fractional calculus operators," Integral Transforms and Special Functions, vol. 15, no. 1, pp. 31-49, 2004.

[4] C.-Y. Ma, B. Shiri, G.-C. Wu, and D. Baleanu, "New fractional signal smoothing equations with short memory and variable order," Optik, vol. 218, Article ID 164507, 2020.

[5] I. Podlubny, Fractional Differential Equations: An Introduction to Fractional Derivatives, Fractional Differential Equations, to Methods of their Solution and Some of their Applications, Elsevier, Amsterdam, Netherlands, 1998.

[6] G. Yang, B. Shiri, H. Kong, and G. C. Wu, "Intermediate value problems for fractional differential equations," Computational and Applied Mathematics, vol. 40, no. 6, pp. 1-20, 2021.

[7] M. Kako, "Nonlinear wave modulation in cold magnetized plasmas," Journal of the Physical Society of Japan, vol. 33, no. 6, pp. 1678-1687, 1972.

[8] B. Shiri, G.-C. Wu, and D. Baleanu, "Collocation methods for terminal value problems of tempered fractional differential equations," Applied Numerical Mathematics, vol. 156, pp. 385-395, 2020.

[9] B. Shiri, G. C. Wu, and D. Baleanu, "Terminal value problems for the nonlinear systems of fractional differential equations," Applied Numerical Mathematics, vol. 170, pp. 162-178, 2021.
[10] T. Xiong, Y. Lv, and W. Yi, "Nonlinear vibration and control of underwater supercavitating vehicles," IEEE Access, vol. 6, pp. 62503-62513, 2018.

[11] D. Lu, A. R. Seadawy, and M. Arshad, "Bright-dark solitary wave and elliptic function solutions of unstable nonlinear Schrödinger equation and their applications," Optical and Quantum Electronics, vol. 50, no. 1, p. 23, 2018.

[12] V. A. Shchepetil'inikov, P. A. Starodubtsev, V. I. Traskovskii, A. G. Senchenko, and R. N. Alifanov, "Method of the finding the nonlinear phenomenas in ocean from moving undersea object and their theoretical explanation," Journal of Siberian Federal University. Mathematics \& Physics, vol. 3, no. 2, pp. 267-75, 2010.

[13] J. Y. Parlange, "Water transport in soils," Annual Review of Fluid Mechanics, vol. 12, no. 1, pp. 77-102, 1980.

[14] A Pimpinelli and J Villain, Physics of Crystal Growth, Cambridge University Press, Cambridge, UK, 1998.

[15] A Ankiewicz, M Bokaeeyan, and N Akhmediev, "Shallowwater rogue waves: an approach based on complex solutions of the Korteweg-de Vries equation," Physical Review E, vol. 99, no. 5, Article ID 050201, 2019.

[16] J. Singh, D. Kumar, and S. Kumar, "A new fractional model of nonlinear shock wave equation arising in flow of gases," Nonlinear Engineering, vol. 3, no. 1, pp. 43-50, 2014.

[17] X.-J. Yang and J. T. Machado, "A new fractional operator of variable order: application in the description of anomalous diffusion," Physica A: Statistical Mechanics and its Applications, vol. 481, pp. 276-83, 2017.

[18] R Arora, M. J. Siddiqui, and V. P. Singh, "Solution of modified equal width wave equation, its variant and non-homogeneous Burgers' equation by RDT method," American Journal of Computational and Applied Mathematics, vol. 1, no. 2, pp. 53-56, 2011.

[19] L. R. T. Gardner and G. A. Gardner, "Solitary waves of the equal width wave equation," Journal of Computational Physics, vol. 101, no. 1, pp. 218-223, 1992.

[20] K. R. Raslan, "Collocation method using quartic B-spline for the equal width (EW) equation," Applied Mathematics and Computation, vol. 168, no. 2, pp. 795-805, 2005.

[21] H. Hamabata and T. Namikawa, "Propagation of hydromagnetic waves in a cold plasma mixed with hot ions," Journal of Plasma Physics, vol. 33, no. 3, pp. 443-453, 1985.

[22] Q. Zhou, A. Sonmezoglu, M. Ekici, and M. Mirzazadeh, "Optical solitons of some fractional differential equations in nonlinear optics," Journal of Modern Optics, vol. 64, no. 21, pp. 2345-2349, 2017.

[23] M. Khater, R. A. Attia, and D. Lu, "Modified auxiliary equation method versus three nonlinear fractional biological models in present explicit wave solutions," Mathematical and Computational Applications, vol. 24, no. 1, p. 1, 2019.

[24] N. Anjum and J.-H. He, "Laplace transform: making the variational iteration method easier," Applied Mathematics Letters, vol. 92, pp. 134-138, 2019.

[25] V. Daftardar-Gejji and H. Jafari, "An iterative method for solving nonlinear functional equations," Journal of Mathematical Analysis and Applications, vol. 316, no. 2, pp. 753-763, 2006.

[26] H. Jafari, "Iterative methods for solving system of fractional differential equations," Doctoral dissertation, Ph. D. Thesis, Pune University, Pune City, India, 2006.

[27] A. Prakash, M. Goyal, and S. Gupta, "Fractional variational iteration method for solving time-fractional Newell-Whitehead-Segel equation," Nonlinear Engineering, vol. 8, no. 1, pp. 164-171, 2019. 
[28] A. Prakash and H. Kaur, "A new numerical method for a fractional model of non-linear Zakharov-Kuznetsov equations via Sumudu transform," Methods of Mathematical Modelling, vol. 189, p. 204, 2019.

[29] S. A. Pasha, Y. Nawaz, and M. S. Arif, "The modified homotopy perturbation method with an auxiliary term for the nonlinear oscillator with discontinuity," Journal of Low Frequency Noise, Vibration and Active Control, vol. 38, no. 34, pp. 1363-1373, 2019.

[30] H. Yépez-Martínez and J. F. Gómez-Aguilar, “A new modified definition of Caputo-Fabrizio fractional-order derivative and their applications to the multi step homotopy analysis method (MHAM)," Journal of Computational and Applied Mathematics, vol. 346, pp. 247-60, 2019.

[31] D. N. Yu, J. H. He, and A. G. Garcia, "Homotopy perturbation method with an auxiliary parameter for nonlinear oscillators," Journal of Low Frequency Noise, Vibration and Active Control, vol. 38, no. 3-4, pp. 1540-54, 2019.

[32] T. R. Prabhakar, A Singular Integral Equation with a Generalized Mittag Leffler Function in the Kernel, University of Delhi, New Delhi, India, 1969.

[33] R. Gorenflo, A. A. Kilbas, F. Mainardi, and S. V. Rogosin, Mittag-Leffler Functions, Related Topics and Applications, Springer, Berlin, Germany, 2014.

[34] P. Miskinis, "The Havriliak-Negami susceptibility as a nonlinear and nonlocal process," Physica Scripta, vol. T136, no. T136, Article ID 014019, 2009.

[35] R. Garrappa, F. Mainardi, and M. Guido, "Models of dielectric relaxation based on completely monotone functions," Fractional Calculus and Applied Analysis, vol. 19, no. 5, pp. 1105-1160, 2016.

[36] A. Stanislavsky and K. Weron, "Atypical case of the dielectric relaxation responses and its fractional kinetic equation," Fractional Calculus and Applied Analysis, vol. 19, no. 1, pp. 212-228, 2016.

[37] A. Giusti and I. Colombaro, "Prabhakar-like fractional viscoelasticity," Communications in Nonlinear Science and Numerical Simulation, vol. 56, pp. 138-143, 2018.

[38] M. D’Ovidio and F. Polito, "Fractional diffusion-telegraph equations and their associated stochastic solutions," Theory of Probability \& Its Applications, vol. 62, no. 4, pp. 552-74, 2018.

[39] K. Górska, A. Horzela, L. Bratek, K. A. Penson, and G. Dattoli, "The probability density function for the Havriliak-Negami relaxation," 2016, https://arxiv.org/abs/1611.06433.

[40] J. An, E. Van Hese, and M. Baes, "Phase-space consistency of stellar dynamical models determined by separable augmented densities," Monthly Notices of the Royal Astronomical Society, vol. 422, no. 1, pp. 652-664, 2012.

[41] L. Beghin and E. Orsingher, "Fractional poisson processes and related planar random motions," Electronic Journal of Probability, vol. 14, pp. 1790-826, 2009.

[42] M. H. Derakhshan and A. Ansari, "On Hyers-Ulam stability of fractional differential equations with Prabhakar derivatives," Analysis, vol. 38, no. 1, pp. 37-46, 2018.

[43] M. H. Derakhshan and A. Ansari, "Numerical approximation to Prabhakar fractional Sturm-Liouville problem," Computational and Applied Mathematics, vol. 38, no. 2, p. 71, 2019.

[44] M. H. Derakhshan, M. Ahmadi Darani, A. Ansari, and R. Khoshsiar Ghaziani, "On asymptotic stability of Prabhakar fractional differential systems," Computational Methods for Differential Equations, vol. 4, no. 4, pp. 276-84, 2016.

[45] A. Liemert, T. Sandev, and H. Kantz, "Generalized Langevin equation with tempered memory kernel," Physica A:
Statistical Mechanics and its Applications, vol. 466, pp. 356369, 2017.

[46] A. Ghorbani, "Beyond adomian polynomials: he polynomials," Chaos, Solitons \& Fractals, vol. 39, no. 3, pp. 1486-1492, 2009.

[47] P. Veeresha, D. G. Prakasha, and D. Kumar, "An efficient technique for nonlinear time-fractional Klein-Fock-Gordon equation," Applied Mathematics and Computation, vol. 364, Article ID 124637, 2020.

[48] M. K. Sadabad, A. J. Akbarfam, and B. Shiri, "A numerical study of eigenvalues and eigenfunctions of fractional SturmLiouville problems via Laplace transform," Indian Journal of Pure and Applied Mathematics, vol. 51, no. 3, pp. 857-868, 2020.

[49] M. Khalid, F. S. Khan, and M. Sultana, "A highly accurate numerical method for solving nonlinear time-fractional differential difference equation," Mathematical Methods in the Applied Sciences, vol. 44, no. 10, pp. 8243-8253, 2019.

[50] S. K. Bhowmik and S. B. Karakoc, "Numerical solutions of the generalized equal width wave equation using the Petrov-Galerkin method," Applicable Analysis, vol. 100, no. 4, pp. 714-734, 2019.

[51] S. B. GaziKarakoc and K. K. Ali, "Analytical and computational approaches on solitary wave solutions of the generalized equal width equation," Applied Mathematics and Computation, vol. 371, Article ID 124933, 2020.

[52] J. Eshaghi, S. Kazem, and H. Adibi, "The local discontinuous Galerkin method for 2D nonlinear time-fractional advectiondiffusion equations," Engineering with Computers, vol. 35, no. 4, pp. 1317-1332, 2019.

[53] F. Jarad, T. Abdeljawad, and Z. Hammouch, "On a class of ordinary differential equations in the frame of AtanganaBaleanu fractional derivative," Chaos, Solitons \& Fractals, vol. 117, pp. 16-20, 2018.

[54] S. L. Shaikh, "Introducing a new integral transform: Sadik transform," American International Journal of Research in Science, Technology, Engineering \& Mathematics, vol. 22, no. 1, pp. 100-102, 2018.

[55] SL Shaikh, "Sadik transform in control theory," International Journal of Innovative Sciences and Research Technology, vol. 3, no. 5, pp. 396-398, 2018.

[56] R. Garra, R. Gorenflo, F. Polito, and Ž. Tomovski, "HilferPrabhakar derivatives and some applications," Applied mathematics and computation, vol. 242, pp. 576-589, 2014.

[57] S. S. Redhwan, S. L. Shaikh, and M. S. Abdo, "Some properties of Sadik transform and its applications of fractional-order dynamical systems in control theory," Advances in the Theory of Nonlinear Analysis and its Application, vol. 4, no. 1, pp. 51-66, 2019.

[58] S. Mashayekhi, Y. Ordokhani, and M. Razzaghi, "A hybrid functions approach for the Duffing equation," Physica Scripta, vol. 88, no. 2, Article ID 025002, 2013. 